\title{
UNIFORM APPROXIMATION BY INCOMPLETE POLYNOMIALS
}

\author{
E. B. SAFF* \\ Department of Mathematics \\ University of South Florida \\ Tampa, Florida 33620 U.S.A.

\section{R. S. VARGA* *} \\ Department of Mathematics \\ Kent State University \\ Kent, Ohio 44242 U.S.A. \\ (Received June 5, 1978)
}

ABSTRACT. For any $\theta$ with $0<\theta<1$, it is known that, for the set of all incomplete polynomials of type $\theta$, i.e., $\left\{p(x)=\sum_{k=s}^{n} a_{k} x^{k}: s \geq \theta \cdot n\right\}$, to have the Weierstrass property on $\left[a_{\theta}, 1\right]$, it is necessary that

$$
\theta^{2} \leq a_{\theta} \leq 1
$$

In this paper, we show that the above inequalities are essentially sufficient as well.

KEY WORDS AND PHRASES. Incomplete polynomials, Weierstrass property, uniform convergence.

AMS (MOS) SUBJECT CLASSIFICATION. $41 \mathrm{~A} 10$ primary; $41 \mathrm{~A} 30$ secondary.

* The research of this author was conducted as a Guggenheim Fellow, visiting at the Oxford University Computing Laboratory, Oxford, England.

$* *$

Research supported in part by the Air Force Office of Scientific Research under Grant AFOSR-74-2729, and by the Department of Energy under Grant EY-76-S-02-2075. 
1. INTRODUCTION.

At the Conference on Rational Approximation with Emphasis on Applications of Padé Approximants, held December 15-17, 1976 in Tampa, Florida, Professor G. G. Lorentz introduced new results and open questions for incomplete polynomials, defined as follows. Let $\theta$ be any given real number with $0 \leq \theta \leq 1$. Then, a real or complex polynomial of the form

$$
p(x)=\sum_{k=s}^{n} a_{k} x^{k},
$$

is said to be an incomplete polynomial of type $\theta$ if $s \geq \theta \cdot n$. Note that the set of all incomplete polynomials of type $\theta$ contains polynomials of arbitrary degree, and that when $\theta>0$, this collection is not closed under ordinary addition. This set, however, is closed under ordinary multiplication.

For such incomplete polynomials, we have, combining recent results,

THEOREM 1.1. (Lorentz [2], and Saff-Varga [4]). For any fixed $\theta$ with $0<\theta \leq 1$, let $\left\{p_{n_{i}}(x)\right\}_{i=1}^{\infty}$ be a sequence of incomplete polynomials of respective types $\theta_{i}$, where $\underset{i \rightarrow \infty}{\lim \inf } \theta_{i} \geq \theta>0$. If

$$
\left|p_{n_{i}}(x)\right| \leq M \text { for all } x \in[0,1] \text {, all } i \geq 1 \text { and } \underset{i \rightarrow \infty}{\lim \operatorname{deg}} p_{n_{i}}=\infty \text {, }
$$

then

$$
p_{n_{i}}(x) \rightarrow 0 \text {, uniformly on every closed subinterval of }\left[0, \theta^{2}\right)
$$

Furthermore, (1.2) is best possible in the sense that, for each $\theta$ with $0<\theta \leq 1$, there is a sequence $\left\{\hat{p}_{n_{i}}(x)\right\}_{i=1}^{\infty}$ of incomplete polynomials of type $\theta$ satisfying $(1.1)$ and a sequence $\left\{\xi_{i}\right\}_{i=1}^{\infty}$ with $\lim _{i \rightarrow \infty} \xi_{i}=\theta^{2}$ for which $\left|\hat{p}_{n_{i}}\left(\xi_{i}\right)\right|=M$ for all $i \geq 1$. Hence, the interval $\left[0, \theta^{2}\right)$ of convergence to zero in (1.2) cannot be replaced by any larger interval $\left[0, \theta^{2}+\epsilon\right)$ for $\epsilon>0$. 
For generalizations of Theorem 1.1, see [4] and [5].

In Lorentz [2], the set of all incomplete polynomials of fixed type $\theta(0<\theta<1)$ is said to have the Weierstrass property on $\left[a_{\theta}, 1\right]$ if, for every continuous function $f$ defined on $\left[a_{\theta}, 1\right]$, there exists a sequence $\left\{p_{n_{i}}(x)\right\}_{i=1}^{\infty}$, with $p_{n_{i}}$ an incomplete polynomial of type $\theta$ for all $i \geq 1$, which converges uniformly to $f$ on $\left[a_{\theta}, 1\right]$. Evidently, from (1.2), a necessary condition that the set of all incomplete polynomials of a fixed type $\theta$, $0<\theta<1$, has the Weierstrass property on $\left[a_{\theta}, 1\right]$ is that

$$
\theta^{2} \leq a_{\theta} \leq 1
$$

The main purpose of this paper is to show that the condition (1.3) is essentially sufficient as well. The outline of the paper is as follows. In $\S 2$, we state our new results and comment on their sharpness and their relation to known results in the literature. The proofs of these new results are then given in $\S 3$.

\section{STATEMENTS OF NEW RESULTS.}

As our first result, we have

THEOREM 2.1. For any fixed $\theta$ with $0<\theta<1$, let $F$ be any continuous function on $[0,1]$ which is not an incomplete polynomial of type $\theta$. Then, a necessary and sufficient condition that $F$ be the uniform limit on $[0,1]$ of a sequence of incomplete polynomials of type $\theta$, is that

$$
F(x)=0 \text { for a11 } 0 \leq x \leq \theta^{2}
$$

As an application of Theorem 2.1, fix any $\theta$ with $0<\theta<1$ and consider any continuous function $\hat{\mathrm{F}}$ on $[0,1]$ with $\|\hat{\mathrm{F}}\|_{L_{\infty}[0,1]}=1$ and with $\hat{\mathrm{F}}$ vanishing on $\left[0, \theta^{2}\right]$ and on $\left[\theta^{2}+\epsilon, 1\right]$, where $0<\epsilon<1-\theta^{2}$. For $\eta>0$, there 
exists, using Theorem 2.1, an incomplete polynomial $\hat{\mathrm{p}}_{\mathrm{n}}$ of type $\theta$ with $\left\|\hat{\mathrm{p}}_{\mathrm{n}}-\hat{\mathrm{F}}\right\|_{L_{\infty}[0,1]}<\eta$, which implies, for $\eta$ sufficiently sma11, that $\hat{\mathrm{p}}_{\mathrm{n}}$ assumes its maximum absolute value on $[0,1]$ in the interval $\left[\theta^{2}, \theta^{2}+\epsilon\right]$. Thus, the sequence $\left\{\left(\hat{p}_{n}(x) /\left\|\hat{p}_{n}\right\|_{L_{\infty}[0,1]}\right)^{j}\right\}_{j=1}^{\infty}$ of incomplete polynomials, each of type $\theta$, cannot tend uniformly to zero in $\left[\theta^{2}, \theta^{2}+\epsilon\right]$ for any $\epsilon$ with $0<\epsilon<1-\theta^{2}$. This observation then gives a different proof of the sharpness portion (cf. [4]) of Theorem 1.1. We also remark that the sufficiency of Theorem 2.1 improves a related result of Roulier [3, Theorem 4] concerning Bernstein polynomials.

From Theorem 2.1, the following is deduced.

THEOREM 2.2. For any $\theta$ with $0<\theta<1$, let $\left\{\theta_{i}\right\}_{i=1}^{\infty}$ be any sequence of real numbers such that $0<\theta_{i}<\theta$ for all $i \geq 1$. Then, for any continuous function $f$ on $\left[\theta^{2}, 1\right]$, there exists a sequence $\left\{P_{n_{i}}(x)\right\}_{i=1}^{\infty}$, with each $P_{n_{i}}$ an incomplete polynomial of type $\theta_{i}$, such that

$$
P_{n_{i}}(x) \rightarrow f(x) \text {, uniformly on }\left[\theta^{2}, 1\right] \text {, }
$$

and such that the sequence $\left\{\mathrm{P}_{\mathrm{n}_{i}}(\mathrm{x})\right\}_{i=1}^{\infty}$ is uniformly bounded on $[0,1]$.

In the case of major interest in Theorem 2.2, i.e., when $\theta_{i} \rightarrow \theta$ as $i \rightarrow \infty$, we remark that the result of Theorem 2.2 is best possible in the following sense. If $[a, b] \supset\left[\theta^{2}, 1\right]$ with $[a, b] \neq\left[\theta^{2}, 1\right]$, then there are, continuous functions on $[\mathrm{a}, \mathrm{b}]$ which cannot be uniformly approximated on $[\mathrm{a}, \mathrm{b}]$ by a sequence $\left\{\mathrm{P}_{\mathrm{n}_{i}}(\mathrm{x})\right\}_{i=1}^{\infty}$, with each $\mathrm{P}_{\mathrm{n}_{i}}$ an incomplete polynomial of type $\theta_{i}$, where $\theta_{i} \rightarrow \theta$ as $i \rightarrow \infty$.

As other consequences of Theorems 2.1 and 2.2, we have

COROLLARY 2.3. For any $\theta$ with $0<\theta<1$, consider any continuous function $\mathrm{f}$ on $\left[\theta^{2}, 1\right]$. Then, for any $\mathrm{q}$ with $1 \leq \mathrm{q}<\infty$, there exists a 
sequence $\left\{P_{n_{i}}(x)\right\}_{i=1}^{\infty}$, with each $P_{n_{i}}$ an incomplete polynomial of type $\theta$, such that

$$
\left\|f-P_{n_{i}}\right\|_{L_{q}\left[\theta^{2}, 1\right]}:=\left\{\int_{\theta^{2}}^{1}\left|f(t)-P_{n_{i}}(t)\right|^{q} d t\right\}^{1 / q} \rightarrow 0 \text { as } i \rightarrow \infty \text {, }
$$

and such that the sequence $\left\{P_{n_{i}}(x)\right\}_{i=1}^{\infty}$ is uniformly bounded on $[0,1]$.

COROLLARY 2.4. For any $\theta$ with $0<\theta<1$, the set of incomplete polynomials of type $\theta$ is dense in the Banach space $L_{q}\left[\theta^{2}, 1\right]$ (with respect to the norm $\|\cdot\|_{L_{q}}\left[\theta^{2}, 1\right]$ ) for each $q$ with $1 \leq q<\infty$.

COROLIARY 2.5. For any $\theta$ with $0<\theta<1$, the set of incomplete polynomials of type $\theta$ is dense in the space of continuous functions on $\left[\theta^{2}+\epsilon, 1\right]$ (with respect to the norm $\|\cdot\|_{L_{\infty}}\left[\theta^{2}+\epsilon, 1\right]$ ) for every $0<\epsilon<1-\theta^{2}$.

The sharpness remarks following Theorem 2.2 similarly apply to the results of Corollaries $2.3-2.5$.

To conclude this section, we remark that Corollary 2.5 leaves as an open question whether or not each continuous function $f$ on $\left[\theta^{2}, 1\right]$ with $f\left(\theta^{2}\right) \neq 0$ is the uniform limit of incomplete polynomials of type $\theta$. In attempting to settle this question, consider the special case of $\theta=\frac{1}{2}$ and $f(x) \equiv 1$ on $\left[\frac{1}{4}, 1\right]$. Setting

$$
\epsilon_{m}:=\inf \left\{\left\|1-x^{m} g_{m}(x)\right\|_{L_{\infty}}\left[\frac{1}{4}, 1\right]: g_{m} \text { is a polynomial of degree } m\right\} \text {, }
$$

a modified Remez algorithm was used to produce the following partial numerical results, rounded to three decimal, where $\alpha_{m}$ denotes the least alternation point in $\left[\frac{1}{4}, 1\right]$ for each $m \geq 1$. 


\begin{tabular}{|l|r|r|}
\hline $\mathrm{m}$ & $\epsilon_{\mathrm{m}}$ & $\alpha_{\mathrm{m}}$ \\
\hline 1 & .220 & .625 \\
\hline 2 & .261 & .494 \\
\hline 3 & .279 & .435 \\
\hline 4 & .289 & .402 \\
\hline 5 & .296 & .380 \\
\hline 6 & .300 & .365 \\
\hline
\end{tabular}

\begin{tabular}{|l|c|c|}
\hline $\mathrm{m}$ & $\epsilon_{\mathrm{m}}$ & $\alpha_{\mathrm{m}}$ \\
\hline 7 & .304 & .353 \\
\hline 8 & .307 & .344 \\
\hline 9 & .309 & .336 \\
\hline 10 & .311 & .330 \\
\hline 11 & .313 & .326 \\
\hline 12 & .314 & .321 \\
\hline 13 & .316 & .317 \\
\hline
\end{tabular}

It is interesting to note that the $\epsilon_{m}$ 's are, in this partial 1 isting, monotone increasing with $\mathrm{m}$.

\section{PROOFS.}

PROOF OF THEOREM 2.1. Let $F$ be any continuous function $[0,1]$ which is not an incomplete polynomial of type $\theta$, and assume that $F$ is the uniform limit of a sequence of incomplete polynomials of type $\theta$. Then, (2.1) follows from (1.2) of Theorem 1.1, establishing the necessity of (2.1). For sufficiency, let $n_{0}$ be any positive integer with $n_{0} \geq(1-\theta)^{-1}$. If $\| y \rrbracket$ denotes the integer part of the real number $y$, let

$$
S_{n}(x):=\sum_{k=\llbracket n \theta \rrbracket}^{n-1} \hat{a}_{k} x^{k} \quad, \quad \forall n \geq n_{0},
$$

be the (unique) least squares approximation to the constant function 1 on $[0,1]$, i.e.,
\[ \sigma_{n}:=\left\{\int_{0}^{1}\left(1-s_{n}(t)\right)^{2} d t\right\}^{\frac{1}{2}}=\inf \left\{\left[\int_{0}^{1}\left(1-\sum_{k=\llbracket n \theta \rrbracket}^{n-1} a_{k} t^{k}\right)^{2} d t\right]^{\frac{1}{2}}: a_{k} \text { is rea } 1\right\} \text {. } \]

Next, set

$$
Q_{n}(x):=\int_{0}^{x} S_{n}(t) d t=\sum_{k=\prod n \theta \rrbracket}^{n-1} \frac{\hat{a}_{k}}{(k+1)} x^{k+1} \quad, \quad \forall n \geq n_{0} .
$$


Note that $Q_{n}$, which is of degree at most $n$, is an incomplete polynomial type $\theta$ for all $n \geq n_{0}$, since $(\llbracket n \theta \mathbb{} n+1) \geq \theta \cdot n$.

From the Müntz theory of best $L_{2}$-approximation on $[0,1]$, it is known (cf. Cheney [1, p. 196]) that

$$
\sigma_{n}=\prod_{j=1}^{n-\prod_{j} n \emptyset}\left(\frac{q_{j}}{1+q_{j}}\right),
$$

where

$$
q_{j}=\llbracket n \theta \rrbracket+j-1, \quad j=1,2, \cdots, n-\llbracket n \theta \rrbracket .
$$

Since the $q_{j}{ }^{\prime} s$ are consecutive integers, the product in (3.3) telescopes to $\llbracket n \theta \rrbracket / n$, whence

$$
\sigma_{n}=\left\{\int_{0}^{1}\left(1-s_{n}(t)\right)^{2} d t\right\}^{\frac{1}{2}}=\frac{\llbracket n \theta \rrbracket}{n} \rightarrow \theta \quad, \quad \text { as } n \rightarrow \infty .
$$

We now show that the sequence $\left\{Q_{n}(x)\right\}_{n=n_{0}}^{\infty}$ converges uniformly to the function $x-\theta^{2}$ on the interval $\left[\theta^{2}, 1\right]$. For this purpose, let $\epsilon$ be an arbitrary real number satisfying $0<\epsilon<\theta^{2}$. From (3.2), we have

$$
x-\theta^{2}-Q_{n}(x)=-\epsilon-Q_{n}\left(\theta^{2}-\epsilon\right)+\int_{\theta^{2}-\epsilon}^{x}\left(1-S_{n}(t)\right) d t,
$$

so that

$$
\left|x-\theta^{2}-Q_{n}(x)\right| \leq \epsilon+\int_{0}^{\theta^{2}-\epsilon}\left|S_{n}(t)\right| d t+\int_{\theta^{2}-\epsilon}^{1}\left|1-S_{n}(t)\right| d t, \forall x \in\left[\theta^{2}, 1\right] .
$$

Applying the Cauchy-Schwarz inequality to the last integral, then

$$
\begin{aligned}
& \left\|x-\theta^{2}-Q_{n}(x)\right\|_{L_{\infty}\left[\theta^{2}, 1\right]} \leq \epsilon+\int_{0}^{\theta^{2}-\epsilon}\left|S_{n}(t)\right| d t+\left(1+\epsilon-\theta^{2}\right)^{\frac{1}{2}} \cdot\left\{\int_{\theta^{2}-\epsilon}^{1}\left(1-S_{n}\right)^{2} d t\right\}^{\frac{1}{2}} \\
& .
\end{aligned}
$$

for a11 $n \geq n_{0}$. Clearly, since $\sigma_{n}=\left\|1-S_{n}\right\|_{L_{2}}[0,1] \rightarrow \theta$ as $n \rightarrow \infty$ from (3.4), 
it follows that there is a constant $M$ such that

$$
\left\|s_{n}\right\|_{L_{2}}[0,1] \leq M \quad, \quad \forall n \geq n_{0} .
$$

Next, note that each $S_{n}(x)$ from (3.1) is an incomplete polynomial of

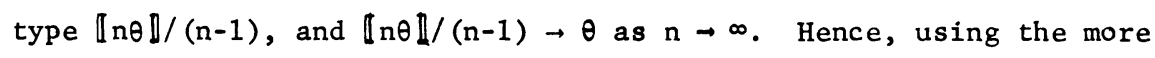
general $\mathrm{L}_{2}$-version of Theorem 1.1 (cf. Saff and Varga [4, Thm. 2.2 and the discussion of $\left.\left(2.4^{\prime \prime}\right)\right]$ ) gives that

$$
S_{n}(x) \rightarrow 0 \text { uniformly on }\left[0, \theta^{2}-\epsilon\right] \text {, as } n \rightarrow \infty \text {. }
$$

Furthermore, on writing

$$
\int_{\theta^{2}-\epsilon}^{1}\left(1-s_{n}(t)\right)^{2} d t=\int_{0}^{1}\left(1-s_{n}(t)\right)^{2} d t-\int_{0}^{\theta^{2}-\epsilon}\left(1-s_{n}(t)\right)^{2} d t
$$

and applying (3.4) and (3.6), we obtain

$$
\lim _{n \rightarrow \infty} \int_{\theta^{2}}^{1}\left(1-S_{n}(t)\right)^{2} d t=\theta^{2}-\left(\theta^{2}-\epsilon\right)=\epsilon .
$$

Consequent1y, from (3.5)-(3.7).

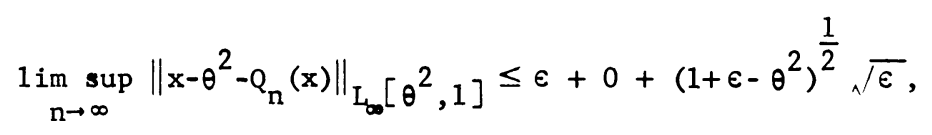

and as $€$ was arbitrary, then

$$
\lim _{n \rightarrow \infty}\left\|x-\theta^{2}-Q_{n}(x)\right\|_{L_{\infty}\left[\theta^{2}, 1\right]}=0 .
$$

We next show that $Q_{n}(x) \rightarrow 0$ uniformly on $\left[0, \theta^{2}\right]$. For any $x$ with $0 \leq x \leq \theta^{2}$, it follows trom the definition of $Q_{n}$ in (3.2) and the Cauchy-Schwarz inequality thet 
whence

$$
\begin{aligned}
\left|Q_{n}(x)\right| & =\left|\int_{0}^{x} s_{n}(t) d t\right| \leq \int_{0}^{x}\left|s_{n}(t)\right| d t \leq \int_{0}^{\theta^{2}}\left|s_{n}(t)\right| d t \\
& \leq \theta \cdot\left\{\int_{0}^{\theta^{2}} s_{n}^{2}(t) d t\right\}^{\frac{1}{2}}, \quad \forall x \in\left[0, \theta^{2}\right],
\end{aligned}
$$

$$
\left(\left\|Q_{n}\right\|_{L_{\infty}}\left[0, \theta^{2}\right]\right)^{2} \leq \theta^{2} \cdot \int_{0}^{\theta^{2}} s_{n}^{2}(t) d t
$$

But

$\int_{0}^{\theta^{2}} s_{n}^{2}(t) d t=\int_{0}^{\theta^{2}}\left[\left(1-s_{n}(t)\right)^{2}-1+2 s_{n}(t)\right] d t=\int_{0}^{\theta^{2}}\left(1-s_{n}(t)\right)^{2} d t-\theta^{2}+2 \int_{0}^{\theta^{2}} s_{n}(t) d t$, and as the last integral is just $2 Q_{n}\left(\theta^{2}\right)$ from (3.2), then

$$
\int_{0}^{\theta^{2}} s_{n}^{2}(t) d t \leq \int_{0}^{1}\left(1-s_{n}\right)^{2} d t-\theta^{2}+2 Q_{n}\left(\theta^{2}\right) \text {. }
$$

Since $\int_{0}^{1}\left(1-S_{n}(t)\right)^{2} d t-\theta^{2} \rightarrow 0$ as $n \rightarrow \infty$ from (3.4) and since $Q_{n}\left(\theta^{2}\right) \rightarrow 0$ as $\mathrm{n} \rightarrow \infty$ from (3.8), it follows from (3.9) and (3.10) that

$$
\lim _{n \rightarrow \infty}\left\|Q_{n}\right\|_{L_{\infty}}\left[0, \theta^{2}\right]=0
$$

Thus, on defining the continuous function $L$ on $[0,1]$ by

$$
L(x):=\left\{\begin{array}{cc}
0 & 0 \leq x \leq \theta^{2}, \\
x-\theta^{2}, & \theta^{2} \leq x \leq 1,
\end{array}\right.
$$

we see from (3.8) and (3.11) that

$$
\lim _{n \rightarrow \infty}\left\|L(x)-Q_{n}(x)\right\|_{L_{\infty}[0,1]}=0
$$

To extend (3.12), we next assert that any continuous function $G(x)$ on $[0,1]$ with 


$$
G(x):= \begin{cases}0, & 0 \leq x \leq \theta^{2}, \\ P(x), & \theta^{2} \leq x \leq 1, \text { where } P \text { is any polynomial } \\ & \text { with } P\left(\theta^{2}\right)=0,\end{cases}
$$

can be uniformly approximated on $[0,1]$ by incomplete polynomials of type $\theta$. Because $P\left(\theta^{2}\right)=0$, we can write

$$
P(x)=\sum_{k=0}^{m} b_{k} x^{k}\left(x-\theta^{2}\right) .
$$

Setting

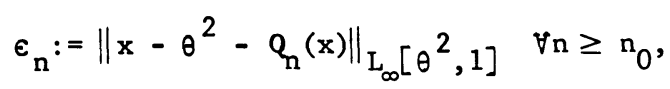

it follows that

$$
\left\|x^{k}\left(x-\theta^{2}-Q_{n}(x)\right)\right\|_{L_{\infty}\left[\theta^{2}, 1\right]} \leq \epsilon_{n}, k=0,1,2, \cdots, \forall n \geq n_{0} .
$$

Next, set $B:=\max \left\{\left|b_{k}\right|: 0 \leq k \leq m\right\}$. Since the case $B=0$ of our assertion is trivial, assume $B>0$ and let $\delta$ be an arbitrary positive number. Since $\epsilon_{\mathrm{n}} \rightarrow 0$ as $\mathrm{n} \rightarrow \infty$ from (3.8), there exists a positive integer $N \geq n_{0}$ such that

$$
\epsilon_{\mathrm{n}} \leq \frac{\delta}{(\mathrm{m}+1) \mathrm{B}} \quad \forall \mathrm{n} \geq \mathrm{N}
$$

Then, for the polynomial $P(x)$ of (3.14), we have from (3.15) and (3.16) that

$$
\left.\begin{array}{rl}
\left\|P(x)-\sum_{k=0}^{m} b_{k} x^{k} Q_{N+m-k}(x)\right\|_{L_{\infty}\left[\theta^{2}, 1\right]} & =\left\|\sum_{k=0}^{m} b_{k} x^{k}\left\{\left(x-\theta^{2}\right)-Q_{N+m-k}(x)\right\}\right\|_{L_{\infty}\left[\theta^{2}, 1\right]} \\
& \leq \sum_{k=0}^{m}\left|b_{k}\right| \epsilon_{N+m-k} \leq \sum_{k=0}^{m}\left|b_{k}\right|\left\{\frac{\delta}{(m+1) B}\right\} \leq \delta .
\end{array}\right\}
$$


Next, we claim that $R(x):=\sum_{k=0}^{m} b_{k} x^{k} Q_{N+m-k}(x)$ is an incomplete polynomial of type $\theta$. Indeed, its degree is at most $N+m$, and as $Q_{N+m-k}(x)$ is an incomplete polynomial of type $\theta$, then each product $x^{k} Q_{N+m-k}(x)$ in this sum has a zero at $\mathrm{x}=0$ of order at least $\mathrm{k}+(\mathrm{N}+\mathrm{m}-\mathrm{k}) \theta$. But as $k+(N+m-k) \theta=(N+m) \theta+k(1-\theta) \geq(N+m) \theta$, then $R(x)$ is an incomplete polynomial of type $\theta$. Thus, as $\delta>0$ was arbitrary, it follows from (3.17) that any polynomial $\mathrm{P}(\mathrm{x})$ with $\mathrm{P}\left(\theta^{2}\right)=0$ can be uniformly approximated on $\left[\theta^{2}, 1\right]$ by a sequence of incomplete polynomials of type $\theta$. Next, as it is evident from (3.11) that

$$
\lim _{\mathrm{N} \rightarrow \infty}\left\|\sum_{k=0}^{m} b_{k} x^{k} Q_{N+m-k}(x)\right\|_{L_{\infty}\left[0, \theta^{2}\right]}=0,
$$

then $G(x)$ of (3.13) can be uniformly approximated on $[0,1]$ by a sequence of incomplete polynomials of type $\theta$.

Now, for an arbitrary function $F(x)$, continuous on $[0,1]$ with $F(x) \equiv 0$ on $\left[0, \theta^{2}\right]$, let $u_{n}(x)$ be the polynomial of degree $n$ of best uniform approximation to $F$ on $\left[\theta^{2}, 1\right]$. If $E_{n}:\left\|F-u_{n}\right\|_{L_{\infty}}\left[\theta^{2}, 1\right]$, then $E_{n} \rightarrow 0$ as $n \rightarrow \infty$. Clearly, $\left|u_{n}\left(\theta^{2}\right)\right|=\left|u_{n}\left(\theta^{2}\right)-F\left(\theta^{2}\right)\right| \leq E_{n}$, whence

$$
\left\|F(x)-\left(u_{n}(x)-u_{n}\left(\theta^{2}\right)\right)\right\|_{L_{\infty}\left[\theta^{2}, 1\right]} \leq 2 E_{n}, \quad \forall n \geq 0 \text {. }
$$

Since $\left(u_{n}(x)-u_{n}\left(\theta^{2}\right)\right)$ is a polynomial vanishing at $\theta^{2}$, call $U_{n}(x)$ its continuous extension to $[0,1]$ with $U_{n}(x) \equiv 0$ on $\left[0, \theta^{2}\right]$ for all $n \geq 0$. Thus, from (3.18),

$$
\left\|F-U_{n}\right\|_{L_{\infty}}[0,1] \leq 2 E_{n} \quad \forall n \geq 0
$$

The previous discussion shows that there is an incomplete polynomial $P_{n}$ of type $\theta$, for every $n>0$, such that

$$
\left\|U_{n}-P_{n}\right\|_{L_{\infty}}[0,1] \leq \frac{1}{n}
$$


whence, with (3.19),

$$
\left\|F-P_{n}\right\|_{L_{\infty}}[0,1] \leq 2 E_{n}+\frac{1}{n}, \quad \forall n>0
$$

Since $E_{n} \rightarrow 0$ as $n \rightarrow \infty$, this proves (cf. (2.1)) that $F(x)$ can be uniformly approximated on $[0,1]$ by $\left\{P_{n}(x)\right\}_{n=0}^{\infty}$, where each $P_{n}(x)$ is an incomplete polynomial of type $\theta$.

PROOF OF THEOREM 2.2. Consider any continuous function $f(x)$ on $\left[\theta^{2}, 1\right]$. Since $\left\{\theta_{n}\right\}_{n=0}^{\infty}$ is any sequence of real numbers with $0<\theta_{n}<\theta$ for all $n \geq 0$, extend $f$ continuously for each $n$ to $[0,1]$, by means of

$$
f_{n}(x):=\left\{\begin{array}{cl}
f(x), & x \in\left[\theta^{2}, 1\right], \\
f\left(\theta^{2}\right)\left(x-\theta_{n}^{2}\right) /\left(\theta^{2}-\theta_{n}^{2}\right), & x \in\left[\theta_{n}^{2}, \theta^{2}\right], \\
0, & x \in\left[0, \theta_{n}^{2}\right] .
\end{array}\right.
$$

Note that $\left\|f_{n}\right\|_{L_{\infty}}[0,1]=\|f\|_{\left[\theta^{2}, 1\right]}$ for all $n \geq 0$, and that each $f_{n}$ satisfies the hypotheses of Theorem 2.1 with $\theta=\theta_{n}$. Applying Theorem 2.1, for any sequence $\left\{\eta_{n}\right\}_{n=0}^{\infty}$ with $\eta_{n}>0$ for all $n \geq 0$ and $\lim _{n \rightarrow \infty} \eta_{n}=0$, there is an incomplete polynomial $p_{n}(x)$ of type $\theta_{n}$ such that

$$
\left\|f_{n}-p_{n}\right\|_{L_{\infty}}[0,1] \leq \eta_{n} \quad \forall n \geq 0
$$

which implies that

$$
\left\|f-p_{n}\right\|_{L_{\infty}}\left[\theta^{2}, 1\right] \leq\left\|f_{n}-p_{n}\right\|_{L_{\infty}}[0,1] \leq \eta_{n} \quad \forall n \geq 0
$$

Consequently, (2.2) holds. It a1so follows from (3.21) that

$$
\left\|p_{n}\right\|_{L_{\infty}}[0,1] \leq\left\|f_{n}\right\|_{L_{\infty}[0,1]}+\eta_{n} \leq\|f\|_{L_{\infty}\left[\theta^{2}, 1\right]}+\eta_{n} \quad \forall n \geq 0,
$$

so that $\left\{p_{n}\right\}_{n=0}^{\infty}$ are uniformly bounded on $[0,1]$. 
To prove the sharpness of Theorem 2.2 , let $[a, b] \supset\left[\theta^{2}, 1\right]$ with $[a, b] \neq\left[\theta^{2}, 1\right]$, take $f(x) \equiv 1$, and suppose there exists a sequence $\left\{P_{n_{i}}(x)\right\}_{i=1}^{\infty}$ of incomplete polynomials of respective types $\theta_{i}$, where $\dot{\theta}_{i} \rightarrow \dot{\sigma}$, such that $P_{n_{i}}(x) \rightarrow f(x)$ uniformly on $[a, b]$. Clearly, $\left\{P_{n_{i}}(x)\right\}_{i=1}^{\infty}$ is uniformly bounded on $[a, b]$. If $0<a<\theta^{2}$, then from $[5, \operatorname{Prop} .1]$, this sequence is necessarily uniformly bounded on $[0,1]$ since $\theta_{i} \rightarrow \theta$. But then, by Theorem 1.1, $P_{n_{i}}(a) \rightarrow 0 \neq f(a)$. Similarly, if $b>1$, we deduce by rescaling that $P_{n_{i}}\left(\theta^{2}\right) \rightarrow 0 \neq f\left(\theta^{2}\right)$.

PROOF OF COROLLARY 2.3. For any sequence $\left\{\eta_{n}\right\}_{n=0}^{\infty}$ with $r_{n}>0$ for d 11 $\mathrm{n} \geq 0$ and $\lim _{n \rightarrow \infty} \eta_{n}=0$, and for any fixed $q$ with $1 \leq q<\infty$, choose in with $\theta^{2}+\delta_{n}^{n \rightarrow \infty} \leq 1$ such that $2\|f\|_{L_{\infty}\left[\theta^{2}, 1\right]} \cdot \delta_{n}^{1 / q}<\eta_{n} / 2$, for every $n \geq 0$. in, define $f_{n}$ on $[0,1]$ by means of

$$
f_{n}(x):= \begin{cases}f(x), & x \in\left[\theta^{2}+\delta_{n}, 1\right], \\ f\left(\theta^{2}+\delta_{n}\right) \cdot\left(x-\theta^{2}\right) / \delta_{n}, & x \in\left[\theta^{2}, \theta^{2}+\delta_{n}\right], \\ 0, & x \in\left[0, \theta^{2}\right],\end{cases}
$$

so that $f_{n}$ is continuous on $[0,1]$ and satisfies the hypotheses of Theorem 2.1. Note, moreover, that $\left\|f_{n}\right\|_{L_{\infty}}[0,1] \leq\|f\|_{L_{\infty}}\left[\theta^{2}, 1\right]$. Now, $\left\|f-f_{n}\right\|_{L_{q}\left[\theta^{2}, 1\right]}=\left\{\int_{\theta^{2}}^{\theta^{2}+\delta_{n}}\left|f(t)-f_{n}(t)\right|^{q} d t\right\}^{1 / q} \leq 2\|f\|_{L_{\infty}\left[\theta^{2}, 1\right]} \cdot \delta_{n}^{1 / q}<\eta_{n} / 2$. Applying Theorem 2.1 to $f_{n}$, there is an incomplete polynomial $P_{n}$ of type $\theta$ such that $\left\|f_{n}-P_{n}\right\|_{L_{\infty}}[0,1]<\eta_{n} / 2$, which also implies that $\left\|f_{n}-P_{n}\right\|_{L_{q}}\left[\theta^{2}, 1\right]<\eta_{n} / 2$. Thus, by the triangle inequality, $\left\|f-P_{n}\right\|_{L_{q}}\left[\theta^{2}, 1\right]<\eta_{n}$, proving (2.3). Moreover, since $\left\|P_{n}\right\|_{L_{\infty}}[0,1] \leq\left\|f_{n}-P_{n}\right\|_{L_{\infty}[0,1]}+\left\|f_{n}\right\|_{L_{\infty}}[0,1]<$ $\eta_{n} / 2+\|f\|_{L_{\infty}}\left[\theta^{2}, 1\right]$, it is clear that the sequence $\left\{P_{n}(x)\right\}_{n=0}^{\infty}$ is uniformly bounded on $[0,1]$. 
PROOF OF COROLLARY 2.4. As an abvious consequence of the fact that the continuous functions are dense in $L_{q}\left[\theta^{2}, 1\right]$ for any $q \geq 1$, Corollary 2.4 then follows directly from Theorem 2.1 and Corollary 2.3.

PROOF OF COROLLARY 2.5. With $\theta_{i}:=\theta$ for all $i \geq 1$, simply apply Theorem 2.2 to any continuous function on $\left[\theta^{2}+\epsilon, 1\right]$, where $0<\epsilon \leq 1-\theta^{2}$.

\section{ACKNOWLEDGMENT}

We wish to thank Mr. M. Lachance (University of South Florida) for having made the calculations which produced the numbers in the tables.

\section{REFERENCES}

1. Cheney, E. W. Introduction to Approximation Theory, McGraw-Hill, New York, 1966 .

2. Lorentz, G. G. Approximation by incomplete polynomials (problems and results), Padé and Rational Approximations: Theory and Applications (E. B. Saff and R. S. Varga, eds.), pp. 289-302, Academic Press, Inc., New York, 1977.

3. Roulier, J. A. Permissible bounds on the coefficients of approximating polynomials, J. Approximation Theory $\underline{3}(1970)$, 117-122.

4. Saff, E. B. and R. S. Varga The sharpness of Lorentz's theorem on incomplete polynomials, Trans. Amer. Math. Soc. (to appear).

5. Saff, E. B. and R. S. Varga On incomplete polynomials, Proceedings of the Oberwolfach Conference, Numerische Methoden der Approximationentheorie, (L. Collatz, G. Meinardus, and H. Werner, eds.), held November 14-19, 1977 (to appear). 


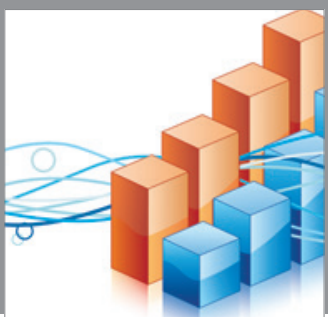

Advances in

Operations Research

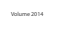

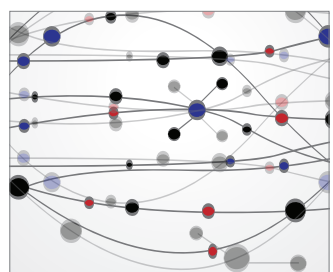

\section{The Scientific} World Journal
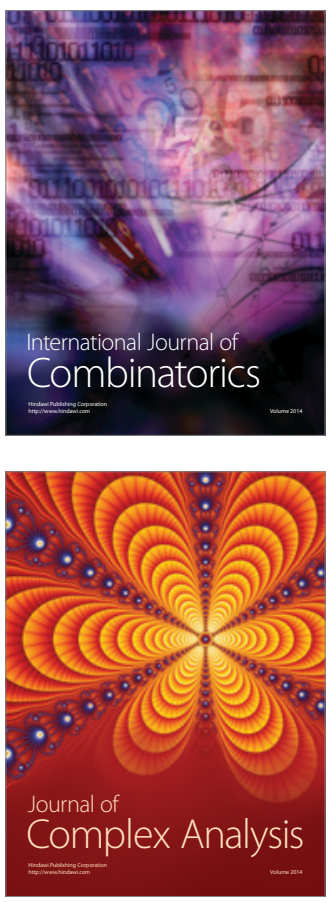

International Journal of

Mathematics and

Mathematical

Sciences
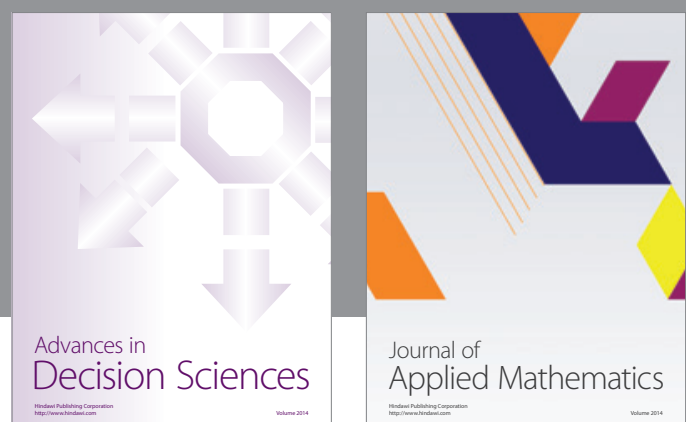

Journal of

Applied Mathematics
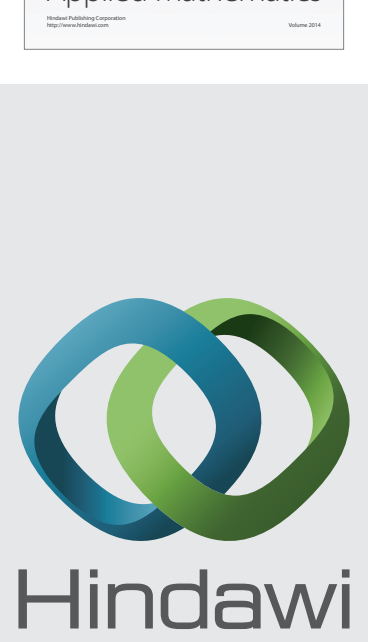

Submit your manuscripts at http://www.hindawi.com
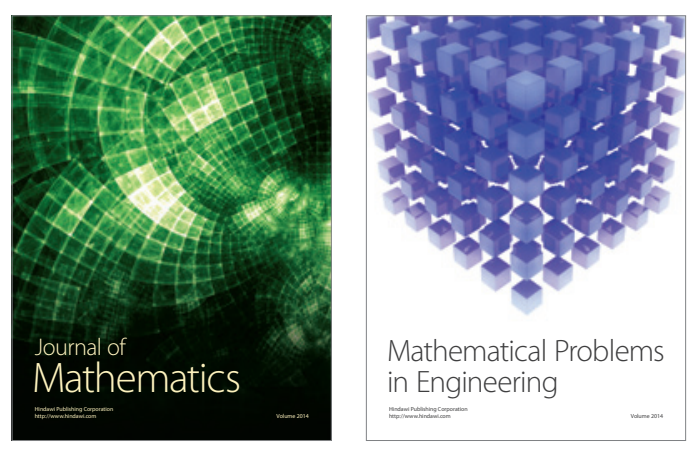

Mathematical Problems in Engineering
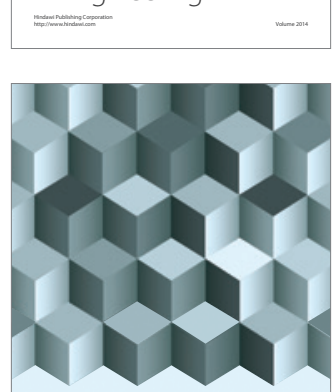

Journal of

Function Spaces
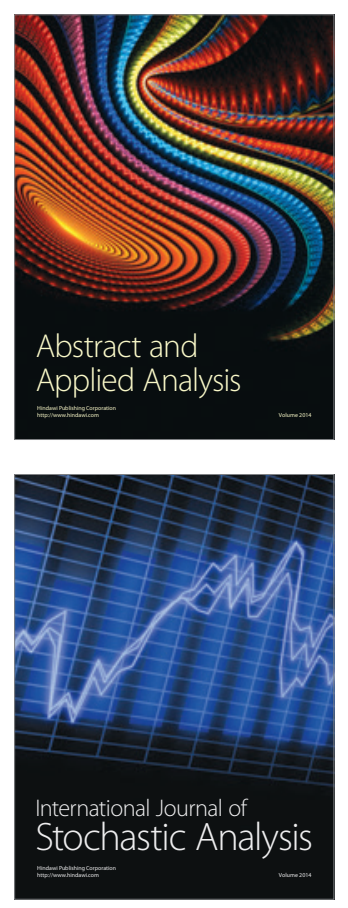

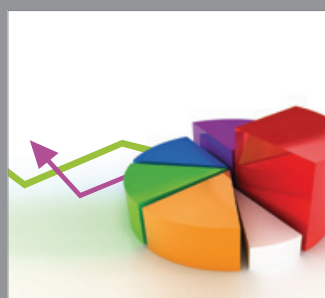

ournal of

Probability and Statistics

Promensencen
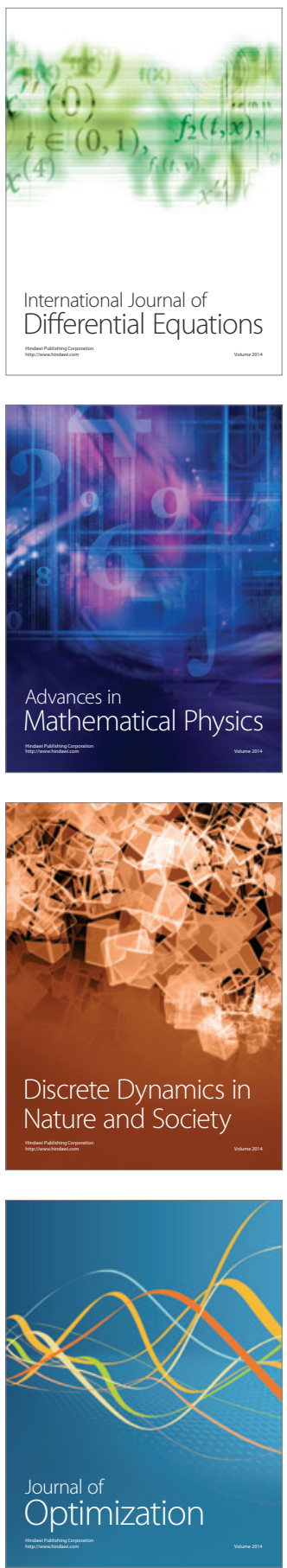